\title{
Role of oral fluorescein in the diagnosis of early papilloedema in children
}

\author{
SUPRIYO GHOSE AND BARUN KUMAR NAYAK
}

From the Paediatric Ophthalmology Service, Dr Rajendra Prasad Centre for Ophthalmic Sciences; All-India Institute of Medical Sciences, Ansari Nagar, New Delhi - 110029 , India

SUMmary Nine eyes of suspected papilloedema, 21 with incipient papilloedema, and 16 with pseudopapilloedema in 23 children aged 1 month to 10 years were examined after oral fluorescein. In pseudopapilloedema the retinal vascular fluorescence and slight disc head fluorescence with sharp margins at 30 minutes markedly declined by 60 minutes. These features were similar to our earlier findings with oral fluorescein in the normal fundi of children. Of the 30 eyes with suspected or incipient papilloedema late disc and peripapillary 'staining', polar or diffuse was observed in 12, the 60 minutes fluorescence being more than at 30 minutes in nine and of equal in intensity in three. In these 12 'positive' eyes, and four more of the 18 'negative' eyes, the retinal vascular fluorescence at 60 minutes was significantly more than at 30 minutes. The problems of interpretation after oral fluorescein in the early diagnosis of papilloedema in children and the possible fallacies are discussed.

There are several conditions in children in which papilloedema is well known, ${ }^{1-4}$ and its early diagnosis is vital in effectively monitoring the treatment and considering timely surgical intervention. In children, however, the early diagnosis of papilloedema is almost entirely dependent on objective features. The ophthalmoscopic diagnosis in 'suspected'2 or even in 'incipient' or 'early's papilloedema may be difficult. Any one sign by itself may be unreliable, and the total picture in its entirety would have to be evaluated before arriving at a clinical opinion. Careful fundus examination, including red-free ophthalmoscopy, ${ }^{\circ}$ fundus photography, and repeated evaluations, ${ }^{6}$ may aid in the early diagnosis. ${ }^{2}$

Fluorescein angiography has always been considered to be valuable in the early detection of papilloedema. ${ }^{1578}$ But the necessity of its conventional intravenous administration usually precludes its wider usage in small and often deformed children. The role of peroral administration of fluorescein dye (oral FA) is gaining importance. " "1" This paper details our experiences of oral fluorescein in children with special reference to the evaluation of its clinical applicability for the early diagnosis of papilloedema.

Correspondence to Dr Supriyo Ghose.

\section{Material and methods}

The procedure adopted by us for oral fluorescein in children has been detailed earlier." The pattern obtained in a few healthy children with normal discs and no detectable fundus abnormality was described.

In the present study 15 children of one month to 10 years of age (Table 1) with the diagnosis of 'suspected' or 'incipient' papilloedema were studied. Of these 15 children 10 had hydrocephalus, three convulsive disorders, one a suspected intracranial space occupying lesion, and one a unilateral proptosis.

The fundus examination was routinely carried out after topical phenylephrine as an outpatient procedure, without sedatives or anaesthesia. ${ }^{2}$ The details

Table 1 Age and sex distribution of the 23 children examined with oral fluorescein

\begin{tabular}{lccc}
\hline Age & $\begin{array}{l}\text { Number of } \\
\text { cases }\end{array}$ & Male & Female \\
\hline 1 month to 11 months & 11 & 9 & 2 \\
1 year to 4 years & 4 & 3 & 1 \\
5 years to 10 years & 8 & 4 & 4 \\
Total & 23 & 16 & 7 \\
\hline
\end{tabular}


Table 2 Disc findings after oral fluorescein in 30 eyes of suspected and early papilloedema in 15 children

\begin{tabular}{|c|c|c|c|c|c|}
\hline \multirow[t]{3}{*}{ Fundus findings } & \multirow[t]{3}{*}{ Group } & \multirow[t]{3}{*}{$\begin{array}{l}\text { No. of } \\
\text { eyes }\end{array}$} & \multicolumn{3}{|c|}{$\begin{array}{l}\text { Late disc staining after ora } \\
\text { fluorescein }\end{array}$} \\
\hline & & & \multirow[t]{2}{*}{ Negative } & \multicolumn{2}{|c|}{ Positive } \\
\hline & & & & Polar & Diffuse \\
\hline $\begin{array}{l}\text { Venous engorgement } \\
\text { (VE) only }\end{array}$ & 1 & $9(5)^{*}$ & $9(5)$ & - & - \\
\hline $\begin{array}{l}\text { VE with blurring of } \\
\text { poles }\end{array}$ & 2 & $17(9)^{*}$ & $9(5) \dagger$ & $6(4) \dagger$ & $2(1)$ \\
\hline $\begin{array}{l}\text { VE with diffuse } \\
\text { blurring of disc } \\
\text { margin }\end{array}$ & 3 & $4(2)$ & - & - & $4(2)$ \\
\hline Total & & $30(15)$ & $18(10)$ & $6(4)$ & $6(3)$ \\
\hline
\end{tabular}

Figures in parentheses denote number of children.

*One patient was observed to have different clinical findings in his two eyes.

†Another patient of group 2 showed polar staining in his right eye,

but no staining could be detected in the left eye.

of the optic nerve head were noted, with special reference to the colour and margins of the disc, the physiological cup, blood vessels, venous pulsations (spontaneous or induced), venous engorgement, venous tortuosity, and the presence of any oedema, haemorrhage, exudates, and sheathing. ${ }^{2}$ Cases of 'developed papilloedema's with obvious oedema with or without haemorrhages were excluded from this study.

The term 'incipient' or 'early' papilloedema' was used to denote the disc picture before significant swelling could be detected. All these discs showed hyperaemia, shallowing or filling up of the cup, mild venous engorgement, and urring of one or both of the upper and lower poles, or diffuse blurring of the disc margins. Twenty-one such eyes in 11 patients formed groups 2 and 3 in this study (Table 2 ).

When only venous engorgement was observed, without or with only minimal blurring of the disc margins, the disc was labelled 'suspected' papilloedema. ${ }^{2}$ Nine such eyes in five children comprised group 1 of this study (Table 2). Except for the 1-month-old infant with unilateral proptosis whose discs showed a faint uniform pallor, all these eyes had hyperaemic discs in addition to the venous engorgement.

Sixteen eyes in eight more children below 10 years of age (Table 1) with a primary clinical diagnosis of pseudopapilloedema were similarly investigated by oral fluorescein. Though mild venous engorgement and hyperaemia were observed in all these eyes along with indistinct disc margins, ${ }^{7}$ the nerve fibre pattern at the margins could be discerned quite clearly on careful fundus examination, especially with red-free ophthalmoscopy. ${ }^{6}$ However, as suspected or early papilloedema could not be ruled out clinically, they were also studied with oral fluorescein. Three of these children had convulsive disorders, two complained of persistent headache, two more were hypermetropic, and one was suffering from prolonged fever.

These 16 eyes clinically labelled as pseudopapilloedema and the 30 eyes with suspected or early papilloedema were investigated after oral fluorescein. The optic discs and blood vessels in the posterior fundus were examined by the reduced and brighter illuminations of the Keeler binocular indirect ophthalmoscope (with the blue Wratten filter) initially, and again at 15,30 , and 60 minutes after dye ingestion." The relative intensities of fluorescence, especially at 30 and 60 minutes, in the papillary and peripapillary areas and of the easily visible larger blood vessels were noted. Wherever possible, fundus photographs before and after oral fluorescein were obtained at these time intervals.

\section{Results}

The age and sex distribution of these 23 patients are depicted in Table 1.

In the 16 eyes with pseudopapilloedema, the slight fluorescence of the disc head observed by 30 minutes almost disappeared by 60 minutes (denoted negative) (Fig. 1).

For the 30 eyes of suspected or incipient papilloedema, the disc features in the various groups after oral fluorescein, with basic reference to the late papillary and peripapillary staining, are analysed in Table 2 . The late staining was either diffuse, involving the entire disc margin and surrounding area (Fig. 2 ), or relatively localised to one or both poles of the disc and adjoining areas. The 60 minutes fluorescence was invariably more prominent than or equal to that observed by 30 minutes (denoted positive, Table 2, Fig. 2). In three eyes with polar staining the 60 minutes fluorescence was equal to the 30 minutes intensity.

All the cases positive on oral fluorescein turned out later to have radiographic/CT (computed tomography) evidence of raised intracranial pressure. Cases negative on oral fluorescein had no such evidence, except only one child with hydrocephalus, in whom CT later suggested raised pressure.

In all the 16 eyes with pseudc yapilloedema the fluorescence of the retinal vessels at 60 minutes declined as compared with the $\mathbf{3 0}$ minutes intensity. However, of the 30 eyes with suspected or early papilloedema 12 with positive late disc staining, polar or diffuse (Table 2), showed fluorescence of the retinal vessels at 60 minutes significantly more than at 30 minutes (Fig. 2). This increased vascular fluoresc- 


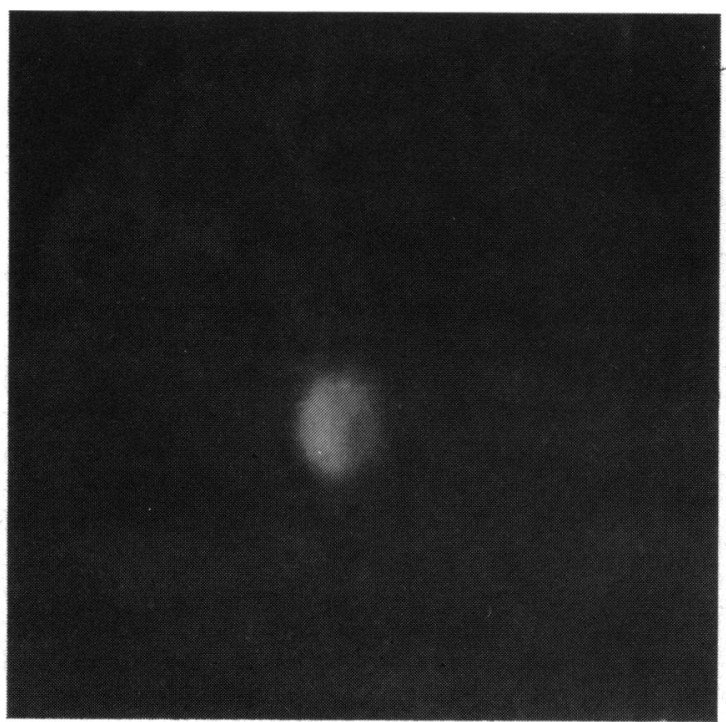

Fig. 1A

ence was also observed in four of the 18 negative eyes (Table 2) with no significant late disc staining.

\section{Discussion}

The fundus features used by us to differentiate clinically between pseudopapilloedema, suspected papilloedema, and incipient or early papilloedema were based on our own past experiences in such children ${ }^{2}$ and on published views. ${ }^{5 \times 12}$

The ophthalmoscopic diagnosis in the early stages may often prove difficult, especially in small children.

\section{Fig. 1C}

Fig. 1 The fluorescein fundus serial photographs after $2 \%$ oral fluorescein at 15,30, and 60 minutes in the right eye of a child with pseudopapilloedema. Note the detectable diffuse fluorescence of the disc and large retinal blood vessels at 15 minutes (A), increasing at 30 minutes (B), and markedly declined at 60 minutes $(\mathrm{C})$. The disc margins are well defined.

It is important to realise that the presence or absence of spontaneous or induced venous pulsations in itself does not decide the diagnosis. ${ }^{2 \times}$ The importance of venous engorgement as a more definite and reliable indicator of early papilloedema is known. ${ }^{258}$ Slight

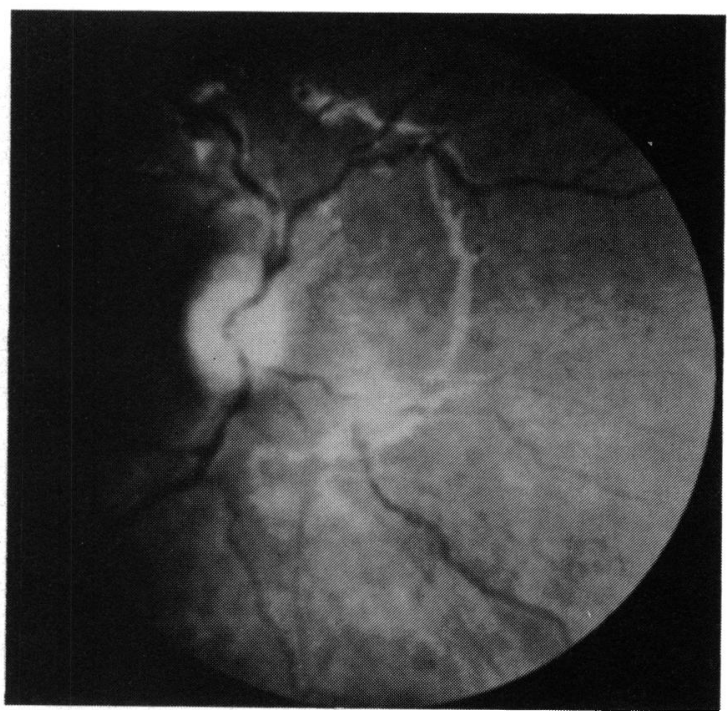

Fig. 2A 


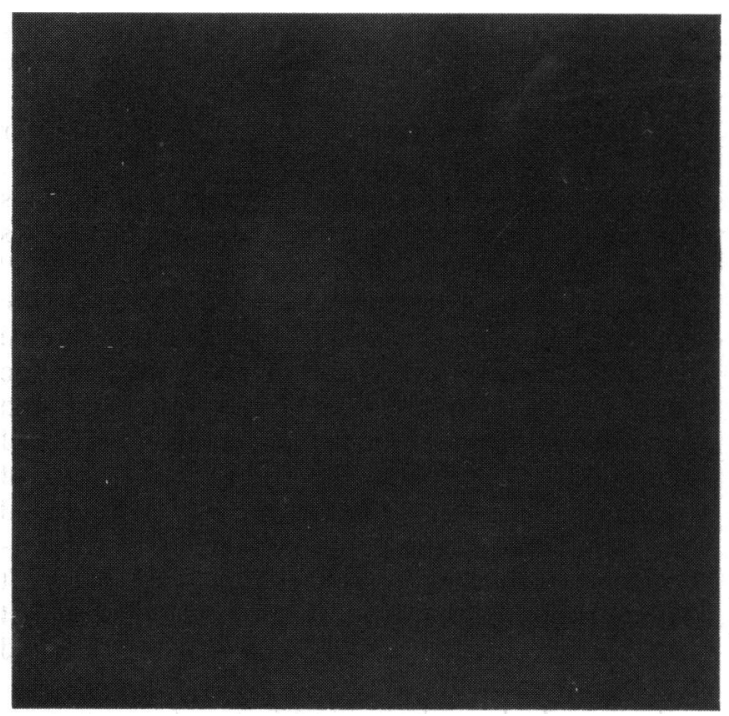

Fig. 2B

Fig. 2 The fundus (A) and fluorescein fundus serial photographs after $1 \%$ oral fluorescein at 15,30, and 60 minutes in the left eye of a child with incipient papilloedema. Note the just detectable disc fluorescence at 15 minutes (B), and better visibility of diffuse disc and retinal vascular fluorescence at 30 minutes $(\mathrm{C})$, increasing to a maximum at 60 minutes with peripapillary staining (D). The right eye at 60 minutes showed a similar picture $(\mathrm{E})$.

blurring of the upper or lower poles or both has been in our experience a very suspicious feature, especially. when an observable venous engorgement is coexistent, even if the other 'typical' features of 'early'

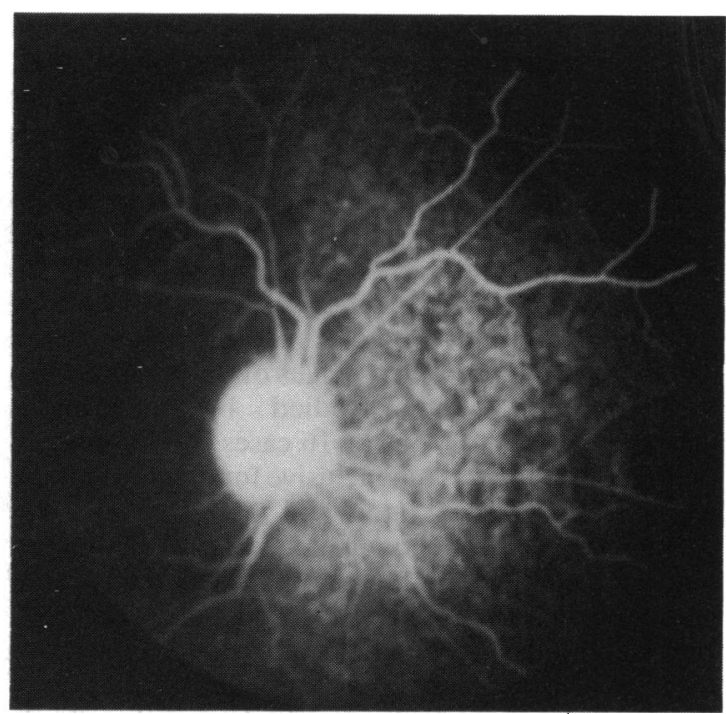

Fig. 2D

papilloedema ${ }^{5}$ are absent; diffuse blurring of the disc margin (usually nasal more than temporal) strongly adds to the clinical suspicion.

In our study venous tortuosity was hardly evident in the earliest stages. Disc hyperaemia was observed in all the eyes, except in the one eye with unilateral proptosis of a 1-month-old infant, where the faint, uniform, bilateral disc pallor appeared to be within normal limits for the age of the child. Hyperaemia was also noted consistently in the discs with pseudopapilloedema. Though the physiological cup was occasionally shallowed, a veil-like greying in this area

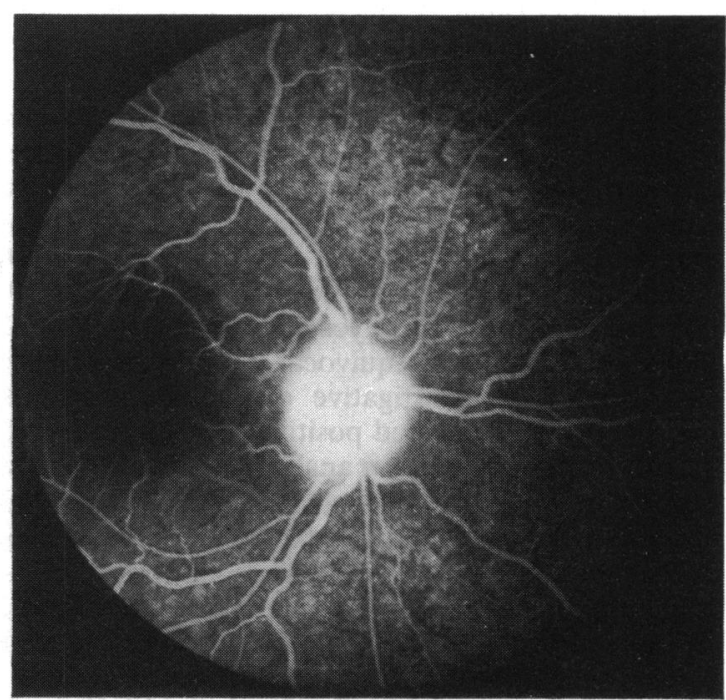

Fig. 2E
Fig. 2C

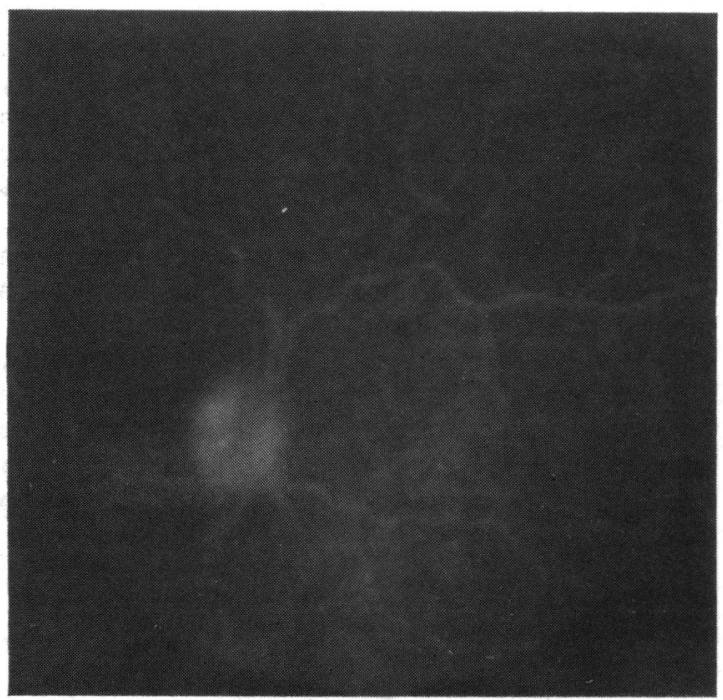


was seldom observed. However, disc hyperaemia and veiling of the tissue at the centre of the disc, though emphasised in the literature as important early signs, ${ }^{5 \times 12}$ do not seem to be as significant as venous engorgement as very early indicators.

Though fluorescein angiography is well known in the differential diagnosis of pseudopapilloedema and papilloedema, ${ }^{7}$ and has also been considered useful in diagnosing early papilloedema, ${ }^{1 \times}$ it has been reported earlier that this is not very satisfactory in the detection of the very early stages of disc oedema. ${ }^{5}$

In another series studied by intravenous fluorescein, ${ }^{13}$ in four out of 16 cases the fluorescein ophthalmoscopy was inconclusive for diagnosis of the papilloedema proved later, and the test in fact was declared negative. Though fluorescein ophthalmoscopy is usually valuable for the differentiation of papilloedema and pseudopapilloedema, even intravenous fluorescein in adults may give a negative result which does not exclude the possibility of papilloedema, ${ }^{13}$ and the method is not entirely reliable. ${ }^{13}$ Fluorescein angiography may fail to distinguish incipient papilloedema in all instances. ${ }^{5}$ Unfortunately no further data seem to be available to prove or disprove this. Hence the early detection of papilloedema by oral fluorescein in small children would obviously be even more problematical.

In our interpretation of the fundus pattern after oral fluorescein from the standpoint of the early diagnosis of papilloedema we were concerned with detecting late 'staining' of the disc and peripapillary areas. In the normal fundi of healthy children the observable fluorescence of the disc head at 30 minutes almost disappeared by 60 minutes." In the six eyes found in the present study to show diffuse late staining (Table 2), the 60 minutes disc fluorescence was consistently more intense than that observed at the 30 minutes phase.

In the six more eyes with late staining relatively localised to one or both poles of the disc and the adjoining areas the 60 minutes fluorescence was either more prominent than or equal in intensity to that noted at 30 minutes. It is significant that the 60 minutes fluorescence was never less than the 30 minutes phase in these eyes. It is possible that in those three eyes with equivocal intensity at 30 and 60 minutes the investigative diagnosis should be interpreted as 'suspected positive', rather than just 'positive' as denoted in our analysis in Table 2. In this connection it is interesting that dye leakage in developing and developed papilloedema has also been noted to be more in the vertical disc axis. ${ }^{1+}$

In our 16 eyes investigated with the clinical diagnosis of pseudopapilloedema it is significant that the pattern and decline of fluorescence after oral administration were virtually identical with that of normal, with the disc margins appearing quite well defined at 30 minutes and no staining of the peripapillary area.

It must be remembered that fluorescein after administration, either orally or intravenously, becomes tagged to the plasma proteins and hence is detectable only as 'staining' or 'leaks' when it comes out of the circulation with the extravasated plasma, ${ }^{7+}$ a state which appears clinically as concomitant disc swelling. Therefore, 'positive' interpretation of oral fluorescein in early papilloedema on the basis of late papillary staining is dependent only on the disrupted functional integrity of the retinal vascular barrier of the capillaries on the optic nerve head. This stage of pathognomonic staining of the nerve head after oral fluorescein is equivalent to the late phase of conventional intravenous fluorescein angiography. Hence it is quite probable that, in spite of a 'negative' result with fluorescein, it may angioscopically be indeed possible to miss the very earliest stages of papilloedema, which perhaps manifest only as venous engorgement without any detectable transudation of the intravascular fluid into the glial tissues of the disc head. Oral fluorescein with higher dye concentrations repeated over intervals and even intravenous fluorescein may have to be considered to rule out the earliest stages of impaired vascular permeability.

However, it is interesting that in all of the 12 eyes denoted 'positive', and in four of the 18 eyes declared 'negative' for disc staining (Table 2) the vascular fluorescence of the easily observable large retinal vessels at 60 minutes was significantly more than at 30 minutes, in contrast to normal eyes." It is probable that the already existent venous stasis and stagnation of blood circulation" are related to this failure of decline of fluorescence. This situation would be compatible with the initial venous engorgement as a very early indicator, ${ }^{2}$ and yet not allow capillary permeability to be so significant on the disc head as to give rise to detectable late disc staining. Hence these four eyes denoted 'negative' in Table 2 should possibly be interpreted as 'suspected positive'.

In an earlier series a few children had been examined by oral fluorescein angiography, ${ }^{4}$ though none of them was suspected of having papilloedema. Our admittedly short-term study is a preliminary attempt at the evaluation of this problem. A much larger and multicentred study would afford adequate clinical experience of the diagnostic criteria in children by oral fluorescein in this very difficult group of cases.

\section{References}

1 Martyn LJ. In: Harley RD, ed. Pediatric ophthalmology. Philadelphia: Saunders, 1975: 476-8.

2 Ghose S. Optic nerve changes in hydrocephalus. Trans Ophthalmol Soc UK 1983; 103: 217-20. 
3 Ghose S, Mehta U. The Kleeblattschädel (cloverleaf skull) syndrome. Indian J Ophthalmol 1986; 34: 61-6.

4 Kalra V. Ghose S. Optic nerve involvement in tuberculous menigitis. Indian Pediatr 1985: 22: 783-5.

5 Walsh FB, Hoyt WF. Clinical neuro-ophthalmology. 3rd ed. Baltimore: Williams and Wilkins, 1969; 1: 567-90.

6 Hoyt WF, Knight CL. Comparison of congenital disc blurring and incipient papilloedema in red-free light-a photographic study. Invest Ophthalmol Vis Sci 1973; 12: 241-7.

7 Miller SJH, Sanders MD, Ffytche TJ. Fluorescein fundus photography in the detection of early papilloedema and its differentiation from pseudopapilloedema. Lancet 1965; ii: 651-4.

8 Huber A. In: Blodi FC, ed. Eye signs and symptoms in brain tumours. 3rd ed. St Louis: Mosby, 1976: 96-128.
9 Kelley JS, Kincaid M, Hoover RE, MacBeth C. Retinal fluorograms using oral fluorescein. Ophthalmology 1980; 87: 805-11.

10 Azad RV, Nayak BK. Tewari HK, Khosla PK. Oral fluorescein angiograhy. Indian J Ophthalmol 1984; 32: 415-7.

11 Nayak BK, Ghose S. A method for fundus evaluation in children with oral fluorescein. Br J Ophthalmol 1987; 71: 907-9.

12 Duke-Elder S, Scott GI. In Duke-Elder S, ed. System of ophthalmology. London: Kimpton, 1971; 12: 54-8.

13 Bynkc HG, Åberg L. Differentiation of papilloedema from pseudo-papilloedema by fluorescein ophthalmoscopy. Acta Ophthalmol (Kbh) 1970; 48: 752-8.

14 Dollery CT, Hodge JV, Engel M. Studies of the retinal circulation with fluorescein. $\mathrm{Br}$ Med J 1962; ii: 1210-5.

Accepted for publication 20 November 1986. 